\title{
Towards the ideal GMP: Homologous recombination and marker gene excision
}

\author{
Holger Puchta* \\ Institut für Pflanzengenetik und Kulturpflanzenforschung (IPK), Corrensstraße 3, D-06466 Gatersleben, Germany \\ Present address: Institut für Botanik II (Molekularbiologie und Biochemie der Pflanzen), Universität Karlsruhe (TH), Kaiserstraße 12, D-76128 \\ Karlsruhe, Germany
}

\begin{abstract}
Summary
A mayor aim of biotechnology is the establishment of techniques for the precise manipulation of plant genomes. Two major efforts have been undertaken over the last dozen years, one to set up techniques for site-specific alteration of the plant genome via homologous recombination («gene targeting») and the other for the removal of selectable marker genes from transgenic plants. Unfortunately, despite multiple promising approaches that will be shortly described in this review no feasible gene targeting technique has been developed till now for crop plants. In contrast, several alternative procedures have been established successfully to remove selectable markers from plant genomes. Intriguingly besides techniques relying on transposons and site-specific recombinases, recent results indicate that homologous recombination might be a valuable alternative for the excision of marker genes.
\end{abstract}

Key words: chimeric oligonucleotides - gene targeting - site specific recombination transformation transposon

Abbreviations: $\mathrm{DSB}=$ double-strand break. $-\mathrm{NHEJ}=$ Non-homologous end joining. $-\mathrm{HR}=$ homologous recombination

\section{Introduction}

A major focus of plant biotechnology over the last years is the development of improved tools for the genetic modification of crop plants. This takes into account broader concerns raised in the public debate, as well as the concerns of regulators and producers. The urgent need of such techniques is documented by the recent UK guidelines on «Best Practices for

\footnotetext{
* E-mail corresponding author: holger.puchta@bio.uka.de
}

the Design of GM crops, that recommend to minimise the soreign, genetic material in GM crops, and the European Council Directive 2001/18/EC on sthe Deliberate Release into the Environment of Genetically Modified Organisms, that requests to sphase out the use of antibiotic resistance markers that confer resistance to cclinically used antibiotics by 2004. In principle the demands for a transgenic plant of the future are that the respective organism contains as few transgenic DNA as possible, at a well defined locus without any further changes of the genome. Thus techniques for the integration of DNA at any possible genomic position as well 
as techniques for the removal of any transgenic sequences superfluous to the final purpose are at the centre of attention of today's genetic engineering of plant genomes. The review will concentrate on recent developments in both fields.

\section{Gene Targeting}

Two different ways for the integration of DNA molecules into genomes are possible: Either a region of sequence identity between the partners is used (homologous recombination [HR], "gene targeting») or no sequence-specific requirements have to be fulfilled [illegitimate recombination also referred to as non-homologous end joining (NHEJ)]. For the controlled manipulation of genomes $\mathrm{HR}$ is of special interest as the product of the reaction is predictable. In transformation experiments in prokaryotes and lower eukaryotes, such as yeast $\mathrm{HR}$, is predominating. Gene targeting is a powerful tool for the directed «knock out» of genes. Using information obtained from various genome sequencing programs the gene coding for the protein of interest can be modified to render it non-functional. The transformed organism carrying a loss of function mutation can then be analyzed for its phenotype. Furthermore, using this technique it is possible to perform subtle changes, such as the modification of single nucleotides in a gene, an achievement that can not be obtained by random insertion mutagenesis. In higher eukaryotes, including plants DNA integrates, mainly via illegitimate recombination in an undirected, sequence-independent manner. Tremendous efforts have been undertaken to improve gene targeting in animals and the development of a feasible technique for mouse embryonic stem (ES) cells has revolutionised basic, as well as applied research in this area. Thousands of «loss of function, mutations of mouse have been generated by gene targeting in ES cells. Numerous experiments indicate gene targeting frequencies of $10^{-2}$ (one homologous integration event per hundred random integration events) or higher in ES cells (for review see Jasin et al. 1996). Unfortunately, similar improvements have not been achieved for higher plants. However, a number of approaches are being developed at present that might indeed lead to a feasible gene targeting technique within the near future. They are described in brief in the following paragraphs. To obtain a more detailed picture of basic aspects of $\mathrm{HR}$ and gene targeting in plants the reader is recommended to consult recently published reviews (Gorbunova and Levy 1999, Hohn and Puchta 1999, Mengiste and Paszkowski 1999, Oh and May 2001, Puchta 1998a, 2002, Schaefer 2001, Vergunst and Hooykaas 1999).

\section{Targeting experiments in higher plants}

Since the pioneering first report on the targeting of a transgene locus in plants (Paszkowski et al. 1988) no convincing enhancement of the targeting frequency has been achieved. In principle, there are two ways to produce transgenic plants.
Either the DNA is directly transferred into plants cells by electroporation or PEG transformation of protoplasts or by the bombardement of various plant tissues (for details of plant transformation see Potrykus and Spangenberg 1995). Alternatively, Agrobacterium tumefaciens is used as a vector for plant transformation (for recent reviews see Hansen and Chilton 1999, Rossi et al. 1996, Tinland 1996, Zupan et al. 2000). Both direct gene transfer (Paszkowski et al. 1988, Halfter et al. 1992) and Agrobacterium-mediated T-DNA transformation (Offringa et al. 1990, Lee et al. 1990) were applied in the initial studies in which the restoration of selectable marker genes was used to estimate the frequency of gene targeting in plants. In all cases the observed gene targeting frequencies were low, $10^{-4}$ to $10^{-5}$, independent of the plant species (tobacco or Arabidopsis) and the transformation method. Considering the factors that improved gene targeting in mouse, various attempts have been undertaken to improve the ratio of homologous to illegitimate recombination. However neither extending the length of homology in the transferred DNA to up to $22 \mathrm{~kb}$ (Thykjaer et al. 1997) nor including negative selectable markers outside of the homology of the targeting vector to enable selection against random integration (Risseeuw et al. 1997, Gallego et al. 1999, Xiaohui Wang et al. 2001) did result in a significantly higher frequency of gene targeting in plants. A major problem of these experiments was that the reported numbers of targeting events were too small for a statistical significant evaluation of putative improvements of the technique. The situation was complicated by the fact that in some cases, although HR resulted in a restored marker gene, the target locus was not changed in the expected way (Offringa et al. 1993, Risseeuw et al. 1995, Reiss et al. 2000, Xiaohui Wang et al. 2001, Hanin et al. 2001). Either integration of the extrachromosomal DNA into the target locus by a combination of homologous and illegitimate recombination was detected, or the sequence homology was found to be copied from the target locus to the extrachromosomal DNA. This modified sequence was then found integrated elsewhere in the genome («ectopic targeting»). These outcomes are not surprising because the main mechanism of recombination in somatic plant cells is described best by a synthesis-dependent strand annealing (SDSA)-like model of recombination (Rubin and Levy 1997, Puchta 1998 b, 1999; for review see Gorbunova and Levy 1999). It could be demonstrated that DNA ends can react independently, i.e. one end might be repaired by homologous and the other by illegitimate recombination. Therefore, gene targeting experiments in higher plants have to include a detailed Southern analysis of recombinants to identify the desired knock-out, in which both ends of the vector sequence have been integrated via homology into the target locus.

Two cases have been reported in which natural genes, the loss of function can not be selected for, were knocked out in Arabidopsis thaliana by gene targeting after Agrobacteriummediated transformation. One Arabidopsis callus out of 2580 tissue culture transformants could be isolated in which target- 
ing of the TGA3 locus had occurred. However the respective callus was a chimera for the targeted locus and could not be regenerated (Miao and Lam 1995). Two years later a mutant Arabidopsis plant could be obtained by knocking out the AGL5 MADS-box gene. Using vacuum infiltration one targeted event was isolated from 750 transformants (Kempin et al. 1997). For several years no further successful gene targeting experiments using vacuum infiltration were reported and no statistically sound conclusion could be drawn from the reported single event (Puchta 1998 a, Liljegren and Yanofsky 1998). Recently however, a new targeting system based on vacuum infiltration of Arabidopsis using the endogenous protoporphyrinogen oxidase (PPO) gene was set up. Incorporation of two mutations in the gene results in resistance against the herbicide Butafenacil. Using Agrobacterium-mediated vacuum infiltration and a targeting vector carrying these mutations, targeting frequencies in repeated experiments of around $7.2 \times 10^{-4}$ were reported, which is about one in event in 1500 transformations (Hanin et al. 2001). Thus, vacuum infiltration, resulting in transformation of female germ cells (Bechtold et al. 2000, Desfeux et al. 2000, Ye et al. 1999), might result in higher targeting rates than transformation of other plant tissues which might be less active in HR. Although the frequencies are still too low for routine gene targeting experiments, the set up of this system should allow the testing of different strategies to improve gene targeting in plants (see below).

An apparently more efficient variant of gene targeting has been reported recently for Drosophila melanogaster (Rong and Golic 2000, 2001; for detailed discussion see also Kumar and Fladung 2001). In this method the construct for targeting is integrated into the host genome flanked by two recognition sites of a site-specific recombinase and includes a site for a rare cutting restriction endonuclease. By induced expression of the site-specific recombinase a DNA circle is excised from the genome. This circle is then linearized after the restriction enzyme (in this case I-Scel) has been expressed resulting in an «activated» DNA molecule with both ends homologous to the target sequence. In the female germline of Drosophila, gene targeting occurred in about one out of 500 cells. Although questions were raised about the general applicability of the technique (Engels 2000) several groups started to test this strategy in plants. On the one hand the setup of the system seems to be very complex, as beside construction of a donor sequence with sites for recombinase and restriction enzyme, expression cassettes for both enzymes have to be included into the transgene construct or supplied in trans and expressed in a concerted manner. On the other hand, if the reaction occurs in an efficient way in planta, every single seedling should represent a germinal excision event. Thus, by the use of suitable marker genes large numbers of plants can be produced and easily screened.

\section{Chimeraplasty}

The observation that transcription enhances HR led to the speculation that the presence of complementary RNA within oligonucleotides might enhance DNA pairing reactions. Therefore, self-complementary chimeric oligonucleotides that consist of a combination of DNA and RNA sequences were developed (for review see Ye et al. 1998, Rice et al. 2001). Chimeric oligonucleotides consist of a DNA «mutator» region of 5 to 6 nucleotides complementary to the target including a mutation to be introduced into the genome, framed by two 2'O-methyl RNA bridges of 8 to 12 nucleotides that are also complementary to the target locus (O-methylation increases the stability of the RNA against degradation within cells). These regions in turn are flanked by hairpin loops of 3 to 4 thymidine nucleotides each. The hairpins are connected to a DNA region of some 30 nucleotides, fully complementary to the supper, RNA-DNA-RNA strand. The lower strand contains a break that seems to be necessary for the topological interwinding of the chimera into the target DNA. Early experiments in mammals demonstrated that chimeric oligonucleotides can be used to induce single nucleotide mutations in a genomic target sequence in vivo. An astonishingly high chromosomal reversion frequency of up to $20 \%$ was reported (Cole-Strauss et al. 1996). Several reports demonstrated the successful use of chimeric oligonucleotides in mammalian cells. However, a major problem remains: the mutation frequencies differ drastically within and between experiments (e.g. Van der Steege et al. 2001).

Due to the low gene targeting frequencies in plants it was obvious to try to establish the technique for targeted mutation of plant genomes (for review see Oh and May 2001). In tobacco (Beetham et al. 1999) and maize (Zhu et al. 1999) an endogenous gene and a transgene were mutated by chimeric oligonucleotides. In contrast to animal cells, where chimeric oligonucleotides are delivered via cationic liposomes, the chimeric oligonucleotides were delivered into the plant cells by biolistics. In both studies the first enzyme of the biosynthetic pathway of branched chain amino acids was chosen as endogenous gene. In tobacco, this gene is referred to as acetolacetate synthase (ALS). The mutation of certain amino acids in the protein results in a resistant phenotype, which can be selected for by application of herbicides. The strategy was to obtain a selectable, dominant mutation in one gene since both plant species harbor a family of ALS genes. The mutation frequencies obtained were assessed to be around $10^{-4}$ (Zhu et al. 1999, Hohn and Puchta 1999). Surprisingly, the specificity of the reaction was not as precise as expected. In tobacco none of the isolated resistant plant cell lines proved to harbor the expected target mutation. In all cases one nucleotide $5^{\prime}$ to the mismatched nucleotide was changed. As controls indicate that chimeric oligonucleotides do not enhance mutation rates all over the genome, the chimeric oligonucleotides - at least in plants - seem to induce mutation not only at the specific mismatch but within the DNA stretch complementary to the target locus. In maize the desired target mutations were indeed recovered (Zhu et al. 1999) and stably propagated (Zhu et al. 2000). Transgenic model systems using the green fluorescent protein (GFP) as a 


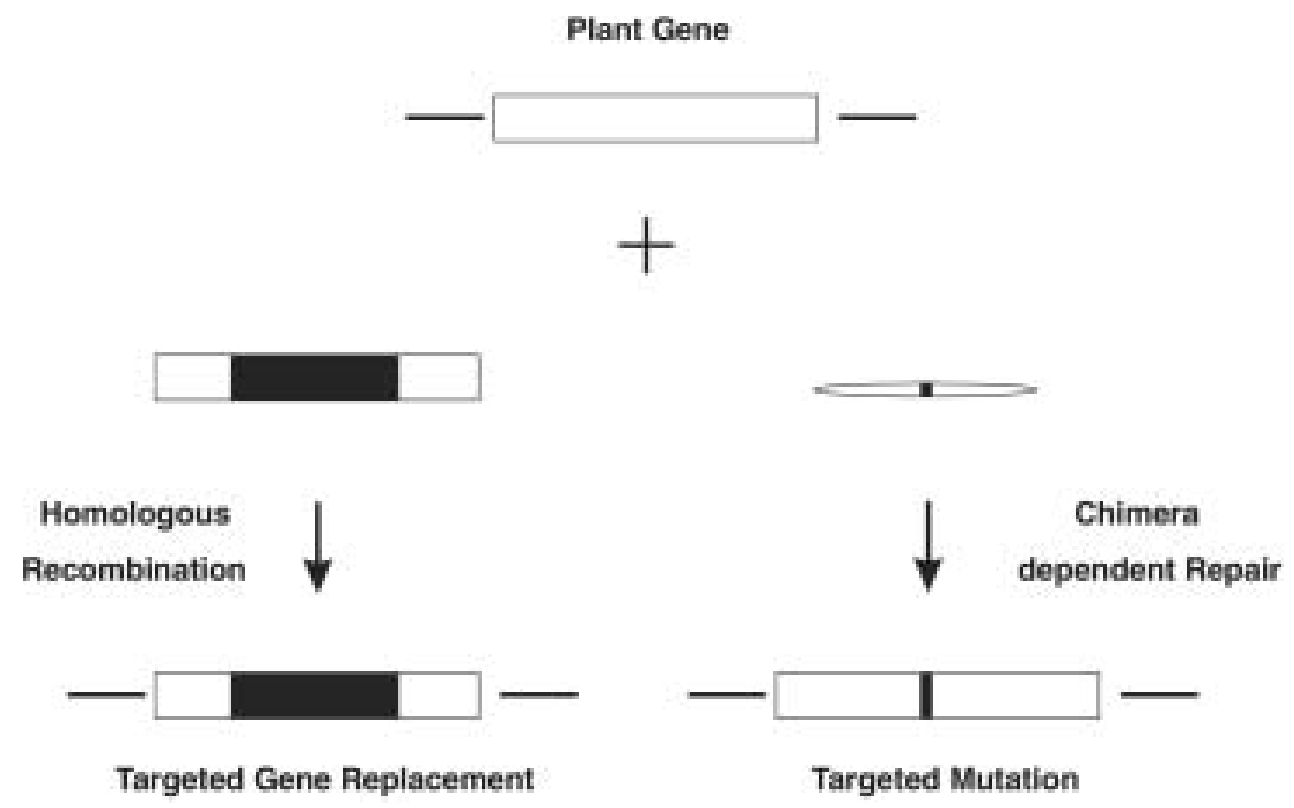

Figure 1. The principle of gene targeting by homologous recombination and the use of chimeric oligonucleotides for the modification of plant genes. In case of HR mutations as well as larger changes (tens of kbs) can be introduced into the target gene. In case of chimera-depended repair only subtle changes of one bp (substitution, insertion or deletion) can be introduced into the target gene [modified after Hohn and Puchta 1999].

marker gene were set up for both plant species. Plants containing an inactive GFP gene were bombarded with the respective chimeric oligonucleotides to reverse the mutations. Putative revertants that are able to express a functional GFP can easily be detected by fluorescence microscopy. In tobacco as well as in maize GFP-positive cells were found at frequencies at least as high as reported for chimeric oligonucleotide-induced mutations in the ALS genes. Taking advantage of the ease of the GFP assay system the establishment of the mutated lines in tobacco and in maize will definitely help to further optimise the efficiency of chimeric oligonucleotide induced mutations in plants.

Experiments were also performed using chimeric oligonucleotides in vitro with extracts of mammalian and, more recently, plant cells. It could be demonstrated with human cell extracts that the repair reaction requires the presence of the mismatch repair protein Msh2. In extracts of a cell line devoid of Msh2 and wild type cell extracts depleted of Msh2 by a specific antibody, a reduced efficiency of repair was measured (Cole-Strauss et al. 1999). Thus, whereas gene targeting relies exclusively on factors involved in $\mathrm{HR}$, chimeric oligonucleotide-directed repair seems to require factors involved in mismatch repair. In line with this argument is the finding that via the use of chimeric oligonucleotides preferentially base substitutions and with less efficiency deletions or insertions of one or two bases in the target locus can be achieved (Ye et al. 1998), whereas via HR genomic sequences of tens of kbs can be inserted or deleted (see Fig. 1). In vitro experiments, performed with nuclear extracts of different plant species, demonstrated that in extracts of tobacco the repair was in some cases less precise than in the extracts of other plant species (Rice et al. 2000). This finding is in accordance with the in vivo data (Beetham et al. 1999). Recent result indicate that with single stranded DNA oligonucleotides with varying numbers of phosophorothioate base analogons at the $3^{\prime}$ and $5^{\prime}$ ends (to block exonucleolitic degradation) even higher repair efficiencies can be achieved than with chimeric oligonucleotides in animal as well as in plant extracts (Gamper et al. 2000). In vivo experiments in yeast demonstrated that phosophorothioate containing oligonucleotides can indeed be used for mutating genes at higher efficiencies than chimeric oligonucleotides (Liu et al. 2001). However the reported frequencies of around $10^{-4}$ are still much too low for practical applications. Therefore further efforts have to be taken to establish a feasible technique for genomic repair in plants.

\section{Production of plants with a hyper-recombination phenotype}

Whereas the approaches described above concentrate on the manipulation of the incoming DNA to obtain an efficient gene targeting frequency, an alternative approach would be to change the genetic background of the host plants. Although this strategy looks promising, one has to keep in mind that a mutated genetic background might lead to genomic instabilities and therefore further efforts are required to avoid unwanted secondary effects due to this background.

A way to change the genetic background in plants is to supplement plants with an enzyme machinery from an orga- 
nism in which HR is efficient. Expressing proteins in tobacco involved in $\mathrm{HR}$ in $E$. coli resulted in two cases in an enhancement of at least certain types of recombination reactions in tobacco plants. Plants transgenic for RecA (Reiss et al. 1996), the key actor of HR in bacteria and for RuvC (Shalev et al. 1999), which is responsible for the resolution of HR intermediates (Holliday junctions) in E. coli were generated. In both cases different kinds of $\mathrm{HR}$ reactions, intrachromosomal recombination and sister chromatid exchange for RecA and intra- and interchromosomal recombination for RuvC, were strongly enhanced in these plants. However, whereas no gene targeting experiments have been reported yet for the RuvC plants, in the respective experiments with RecA plants no significant enhancement of the gene targeting frequency could be achieved with Agrobacterium-mediated transformation (Reiss et al. 2000). The outcome of these experiments clearly show, that it is very difficult to predict the effects of the expression of a heterologous protein on recombination in plants. Due to complex interactions with different factors it seems probable that the respective protein is only able to participate in some but not all of the reactions as in its natural background.

Basic features of the enzyme machinery involved in recombination are conserved between yeast, mammals and plants. Thus, factors of putatively similar function can be identified by sequence homologies (Vergunst and Hooykaas 1999, Bhatt et al. 2001). With the complete sequence of the Arabidopsis genome at hand multiple open reading frames with similarities to genes involved in repair and recombination in other organisms have been identified (Arabidopsis Genome Initiative 2000). It has been reported for vertebrates that mutations in certain genes lead to enhanced HR frequencies. E.g., the knockout of the Mre11 gene of chicken leads to increased gene targeting frequencies (Yamaguchi-Iwai et al. 1999). Mre 11 together with Rad50 is in yeast part of a multifunctional protein complex that can act as nuclease and is involved in homologous as well as in illegitimate recombination (for review see Haber 1998). Homologous factors exist in Arabidopsis (Hartung and Puchta 1999, Gallego et al. 2001) and it was demonstrated that a Rad50 T-DNA insertion mutant showed an increase of intrachromosomal HR by one order of magnitude (Gherbi et al. 2001). It will be interesting to see whether a similar increase can also be detected for gene targeting. There are several candidate genes which, mutated or suppressed, might lead to higher targeting frequencies. In chicken, enhanced target integration of DNA was found for a mutant of the gene responsible for the Bloom's syndrome (BLM) (Wang et al. 2000). BLM is a member of the RecQ helicase family involved in pro- and eukaryotes in DNA replication and recombination (for review see Karow et al. 2000). Recently several RecQ homologues have been characterised in Arabidopsis (Hartung et al. 2000).

Blocking illegitimate recombination should increase the relation of homologous to random integration events. Two components of this pathway from Arabidopsis, DNA Ligase IV and
XRCC4, have already been characterised biochemically (West et al. 2000) and more will follow. Without doubt during the next few years a series of mutants with insertions within different genes involved in recombination processes will be produced and characterised. Analysis of their effects on recombination may lead to identification of plants with enhanced gene targeting frequencies. Alternatively, also overexpression of Arabidopsis genes involved in HR or its regulation might help to increase gene targeting efficiency.

The classical genetic method to use undirected mutagenesis to isolate a phenotype of interest can provide important new insights, because in contrast to gene isolation by homology search, new genes involved in the phenomenon of interest might be found. As defects in recombination should be correlated with an impaired ability to repair DSBs resulting in an increased sensitivity against X-rays, screening of Arabidopsis populations, mutagenized by T-DNA insertions, for radiation-sensitive mutants should lead to the identification of genes involved in this process. Indeed, an insertion mutant into a gene (MIM) closely related to the structural maintenance of chromosomes (SMC) gene family of other eukaryotes was correlated with an X-ray sensitive phenotype. The mutant showed a decreased frequency of intrachromosomal HR (Mengiste et al. 1999) and over-expression of the MIM gene in wild type Arabidopsis plants indeed increased the frequency of homologous intrachromosomal recombination, although the effect was too small to expect a decisive improvement of gene targeting frequencies (Hanin et al. 2000).

A more direct strategy would be to screen for hyper-recombination mutants. In the «sulfur» mutation of tobacco, somatic crossovers between homologues can be detected in planta as yellow/green twin sectors, although the molecular nature of the process is not characterised in detail (Carlson 1974). Using a transposon as insertion mutagen, a plant with interchromosomal recombination enhanced by three orders of magnitude could be isolated. Moreover, the mutant showed increased resistance to gamma irradiation. Interestingly, although the frequency of HR between extrachromosomal substrates was also increased, no enhancement could be detected with an intrachromosomal substrate (Gorbunova et al. 2000). It will be very interesting to test this mutant for gene targeting, especially as an enhancement of several orders of magnitude would indeed be a major break through. Further mutant screens e.g. with intrachromosomal recombination substrates and also with Arabidopsis (Swoboda et al. 1994) should lead to the identification of more hyper-recombining plant mutants. This strategy seems to be a promising approach to reach the goal of setting up a feasible gene targeting technique in higher plants.

\section{Efficient gene targeting in moss}

In contrast to flowering plants, the moss Physcomitrella patens is able to integrate DNA efficiently by HR (for a recent re- 
view see Schaefer 2001). This finding (Schaefer and Zryd 1997) marked a major breakthrough, especially as it could be demonstrated that the technique can indeed be used efficiently for the knock out of gene functions (Strepp et al. 1998, Girke et al. 1998). The moss has turned out as a very valuable system for investigation of basic processes of plant biology (for recent reviews on the biology of Physcomitrella patens see Cove et al. 1997, Reski 1998a, 1999). As most of the ESTs of Physcomitrella patens show strong homology to genes of other plants (e.g. Reski et al. 1998), it seems likely that in many cases homologues of higher plant genes might be found in Physcomitrella. Targeting experiments in Physcomitrella might therefore help to elucidate their general function (Puchta 1998 a, Reski 1998 b). Although efficient gene targeting in moss does not directly help to establish the technique in crop plants it might do so indirectly: It will be important to answer the question why homologous integration is that efficient in moss. Many possible explanations may be considered: 1. Apparently PEG-mediated transformation is especially efficient for gene targeting in moss (Schaefer 2001), but not in higher plants (Mengiste and Paszkowski 1999). 2. Transformation seems to occur mainly during the $\mathrm{G}_{2} / \mathrm{M}$ transition of the cell cycle in moss and it can be speculated that HR is especially efficient at this time (Reski 1998 b). Indirect evidence suggests that in mammals $\mathrm{HR}$ is more efficient during $S$ and $G_{2}$ phase than within $G_{1}$ (Dronkert et al. 2000). However it remains to be proven whether a putative moss-specific cell cycle block is indeed responsible for the dramatic enhancement in HR. 3. It was speculated that the haploid state per $s e$ is a prerequisite for the efficiency of gene targeting (Schaeffer and Zryd 1997), however, in higher plants transformation experiments with haploid tissue did not yield increased gene targeting frequencies (Mengiste and Paszkowski 1999). 4. An intriguing finding is that in many cases several copies of the plasmid DNA were integrated per target locus (Schaeffer and Zryd 1997) and that extrachromosomal DNA can persist in Physcomitrella for longer time periods (Ashton et al. 2000). This hints to the possibility that incoming plasmid DNA might be replicated in the moss and, due to this process, the DNA might become more sactivated for homologous interactions. Only further studies about the recombination mechanisms in Physcomitrella patens and the factors involved will provide better understanding of the recombination behaviour of this organism and support establishing an efficient targeting technology in crop plants.

\section{Marker gene elimination}

An important step during generation of transgenic plants is the identification cells with an integrated transgene within a bulk of non-transformed cells. The classical way to achieve this goal is the use of marker genes within the transgene, the expression of which can be selected for. These markers are mostly conferring antibiotic or herbicide resistance. In recent years concerns were raised - mainly by ecologists and consumer organisations - that the presence of such genes within environment or food might be an unpredictable hazard to the ecosystem as well as to human health. Herbicide resistance genes might be transferred by outcrossing into weeds. The presence of resistance genes against antibiotics in food products might theoretically lead to the spread of these resistances via intestinal bacteria in human populations, although there is no evidence supporting this fact. Therefore, since a row of years studies to avoid marker genes or to eliminate them after use have been conducted and a still growing bunch of methods is becoming available for the elimination of these genes.

In principle, there are four ways to either avoid or get rid of «problematic» selectable marker genes before transgenic plants are brought out into the field: 1. Avoiding selectable marker genes at all. Theoretically, it should be possible to identify among a large number of cells the ones that carry a transgene directly by molecular methods. However, even in the days of automated analysis and polymerase chain reaction such a project is highly demanding and no feasible protocol has been published till now. 2. Use of marker genes that have no possibly «harmful» biological activities. 3. Cotransformation of two transgenes, one carrying the desired trait and the other the selection marker, followed by the segregation of the two. 4. Excision of the selectable marker gene out of the integrated transgene after successful selection by using site specific recombination, transposition or HR. In the following these strategies are briefly described. To obtain a more detailed picture of specific aspects of marker gene elimination in plants the reader is recommended to consult recently published reviews (Puchta 2000, Hohn et al. 2001, Ow 1996, 2001, 2002, Ebinuma et al. 2001, Vergunst and Hooykaas 1999).

\section{Alternative selectable markers}

In parallel to or in combination with marker elimination a new set of markers is being developed. The rational behind this system is that non-transformed cells are not killed as in the procedures using antibiotic - or herbicide resistance genes, rather transformed cells experience a metabolic or developmental advantage. This might even increase the efficiency of regeneration of transformed plants. Of additional value is the non-toxicity of the selective chemicals, as opposed to antibiotics and herbicides. In this respect the bacterial $\beta$-glucuronidase (Joersbo and Okkels 1996), xylose isomerase (Haldrup et al. 1998) and phosphomannose isomerase genes (Joersbo et al. 1998, Negrotto et al. 2000) were used successfully. Also genes coding for enzymes playing a role in hormone metabolism plants as the isopentenyl transferase (ipt) gene from the T-DNA of Agrobacterium were successfully used for the selection of transformants (Ebinuma 1997). The use of a dexamethasone-inducible promoter driving the ipt gene led to the 
recovery of lettuce and tobacco transformants under inducing conditions (Kunkel et al. 1999). By the development of these new selection markers concerns about the spread of herbicide or antibiotic resistance into the environment become obsolete. However, considering reduction of transgene sequences to an absolute minimum as the final aim, the complete elimination of selection markers seems to be more favorable in the long run.

\section{Elimination of marker genes by cotransformation}

One way to separate selectable marker genes from the transgene of interest is to separate them already at the stage of transformation. Regularly Agrobacterium mediated transformation is used for this purpose, as separate integration events occur more regularly using this method than direct gene delivery methods. In principle in cotransformation experiment the desired gene and the transformation marker can be supplied on two T-DNAs within the same binary vector, on two binary vectors within the same Agrobacterium or with two different Agrobacterium strains (Depicker et al. 1985, De Block and Debrouwer 1991, McKnight et al. 1987, Komari et al. 1996). In all cases a fraction of transformants will carry the two transgenes unlinked. Cotransformation frequencies of up to $47 \%$, were reported with a high proportion of both tobacco and rice transformants carrying unlinked transgenes (Komari et al. 1996). This procedure requires fertile plants for genetic separation of the two transgene loci.

\section{Site-specific recombination}

The first demonstration that a selection marker gene can be removed from the genome of a transgenic plant - a milestone in plant biotechnology - was achieved a decade ago. A kanamycin gene placed between two lox sites could be excised from the plant genome by the expression of the Cre recombinase (Dale and Ow 1991; for principle see Fig. 2). The sitespecific recombinase Cre catalyses the circularisation of the genome of the bacteriophage $\mathrm{P} 1$ of $E$. coli using $34 \mathrm{bp}$ long lox-sites at the ends of its genomic unit. By controlled expression of the single chain polypeptide Cre and specific allocation of the lox sites within transgenic constructs, the system can be applied to a set of different genome manipulations. In general, two lox-sites in direct orientation are required for excision of the intervening sequences (Russell et al. 1992). Any kind sequence, e.g. multiple copy transgenes (Srivastava et al. 1999) or genomic sequences, if the lox sites have been moved apart via transposon jumping (Osborne et al. 1995), can be excised via expression of Cre. Inversely a transgene can be integrated site-specifically into a lox site (Albert et al. 1995, Vergunst et al. 1998). Because this reaction is reversible with a bias towards excision, specific lox sites were developed in which after integration the newly combined half sites were no longer functional (Albert et al. 1995). Two lox-sites in inverted orientation are necessary for inversion of the intervening sequence (Medberry et al. 1995). Even the exchange of chromosome arms (Qin et al. 1994) was achieved with the Cre-lox system in plants.

Besides Cre, other single chain recombinases were used for removal of transgene sequences namely the FLP/ftr system of the $2 \mu$ plasmid of $S$. cerevisiae (Lyznik et al. 1996, Kilby et al. 1995) or the R-RS system of the pSR 1 plasmid of Zygosaccharomyces rouxii (Onouchi et al. 1995, Sugita et al. 2000). A common feature of all these systems is that after a first round of transformation transgenic plants are produced that contain between two directly orientated recognition sites of the respective recombinase the sequence to be eliminated. After expression of the single-chain recombinase the recombination reaction is initiated resulting in transgenic plants devoid of the selection marker.

Two developments are taking place in the field recently. Due to the fact that normally a specific site-specific recombinase can only be used for a single round of genome manipulation, because functional sites are left behind in the genome, the interest on «new» site-specific integration systems rose over the years and they are in the process of being introduced into plants (e.g. Thomason et al. 2001). Another economically important goal is the reduction of time required to obtain marker-free transgenic plants. In the early experiments

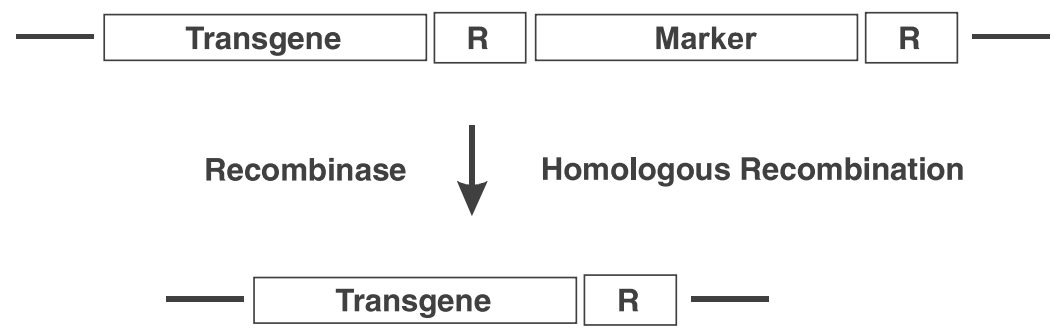

Figure 2. General strategy for the excision of selectable marker genes. Between two identical sequence motives (R) that are recognized by a site-specific recombinase the selectable marker gene is inserted into the transformation vector and used for the selection of transgenic plant cells. After expression of the respective recombinase the marker gene is excised from the plant genome. Alternatively, recombination between the homologous overlaps could also result in marker gene elimination. HR is efficient in chloroplasts and in the nuclear genome if DSB are induced at specific sites within the overlapping region [modified after Puchta 2000]. 

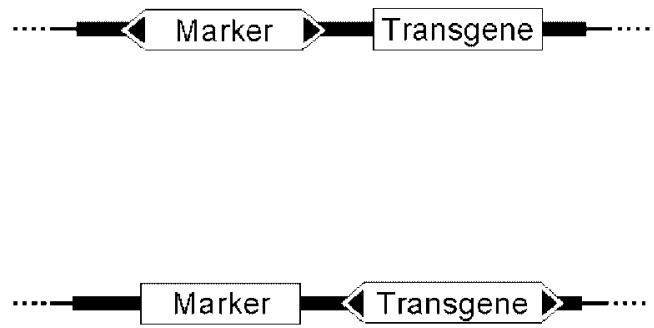
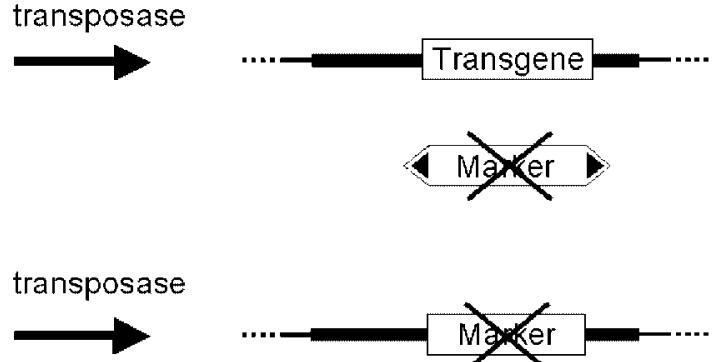

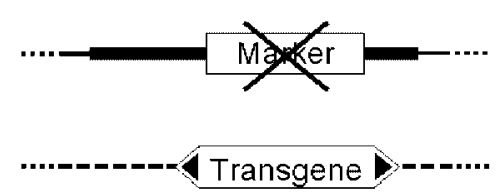

Figure 3. Marker gene elimination using transposable elements. Either the marker or the transgene is flanked by sequence elements required for transposition (black triangles). After expression of the transposase the respective element will be excised from the transgene locus. Thus a marker-free transgene is obtained either in the original transgen position and the marker is eliminated via transposition if no reintegration occurs (upper part) or the transgene integrates in a new position in the genome and the marker has to be eliminated via segregation in the next generation (lower part) [modified after Hohn et al. 2001].

transgenic plants containing the marker were regenerated first, and then crossed to plants expressing the recombinase and finally the progeny of this cross was then checked for marker-free seedlings (Dale and Ow 1991). At present the marker is mostly eliminated soon after transformation within a single tissue culture phase. One approach is to include into the sequence to be deleted from the transforming DNA besides the selection marker a negative selectable marker gene. After transient expression of the recombinase, transgenic plant cells without the marker genes can then be selected for (Gleave et al. 1999). Alternatively, the expression cassette of the recombinase can be put under the control of an inducible promoter and included into the sequence block that will be eliminated from the genome. Using this strategy, after induction of the recombinase, marker-free plants carrying a single copy transgene could be obtained even without the use of counter-selection (Sugita et al. 2000) in one study with astonishingly high frequencies of up to $66 \%$ (Zuo et al. 2001). In addition to the excision of nuclear transgenes in two elegant studies Cre-lox has also been applied recently with high efficiency to excise marker genes residing on a transgen within the chloroplast genome of tobacco (Corneille et al. 2001, Hajdukiewicz et al. 2001).

\section{Elimination of marker-genes by transposases}

Besides site specific recombination, transposable elements can be used to obtain marker-free transgenic plants. The strategy is to connect either the transgen or the selection marker with transposable sequences that the two entities can be moved apart from each other in a controlled reaction after transformation and selection. Both approaches have been applied successfully. In the first one (Fig. 3), the marker gene is placed on a mobile element which after transposition is lost (Gorbunova and Levy 2000). Marker-free transgenic tobacco and aspen plants have been generated at low frequencies by inserting the selectable ipt gene into the transposable element Ac (Ebinuma et al. 1997). The second possibility for transposon-induced dissociation of the marker and the desired gene consists in relocation of the desired gene away from the original transgene locus (Fig. 3). The feasibility of this approach was demonstrated in tomato (Goldsbrough et al. 1993, Yoder and Goldsbrough 1994). The advantage of this system is not only to unlink the marker-gene, but also to create a series of plants with different transgene loci from one original transformant, which is especially appreciated if recalcitrant plants have to be transformed. This re-positioning allows expression of the transgene at different genomic positions and consequently at different levels. However, as segregation of transgene and marker are required and transposons tend to jump into linked positions, this approach is definitely more time consuming than the one described before.

\section{Homologous recombination}

Recently HR became a putative alternative to remove marker genes: Transgenic tobacco calli carrying between two $352 \mathrm{bp}$ long attP-sites a kanamycin gene and a negative selectable marker lost the genes at high frequency during growth. This was surprising since attP-sites are used by the bacteriophage lambda for integration at the attB-site into the E. coli genome, a reaction for which two proteins are needed, the phageencoded integrase (int) and the bacterial integration host factor (IHF). However, none of the proteins were expressed in plants. As we know from various experiments, intrachromosomal HR (ICR) between closely linked repeated sequences in tobacco occurs at frequencies of about $10^{-6}$ (Puchta and Hohn 1996). Therefore the question arises, what could be the cause of the much higher rates. Interestingly only two out of eleven lines showed this high deletion frequencies, that were 
in 3 out of 23 cases associated with HR between the $352 \mathrm{bp}$ long repeats. One is tempted to speculate that the formation of a recombination hot spot at the respective transgene locus might be responsible for the phenomenon (Puchta 2000). This could not only result in preferred integration of transgenes in these loci but afterwards also in further rearrangements within the transgenes. Especially the fact that different kinds of recombination reactions were enhanced to high frequencies is in line with this hypothesis. For a final assessment of the described approach, further experiments on the general applicability of the technique and the stability of the resulting transgenes have to be performed.

Recombination is inducible by DSBs and all current recombination models are based on the repair of such breaks (for review see Paques and Haber 1999). Therefore, it is indeed possible to enhance recombination in plant cells by «activating" specific sites in the genome by the induction of doublestrand breaks (DSB). Via transient expression of the restriction enzyme I-Scel DSBs can be introduced at transgenic restriction sites within plant genome in vivo, resulting in an enhancement of HR as well as of NHEJ by several orders of magnitude (Puchta et al. 1996, Salomon and Puchta 1998, Puchta 1999). Although this strategy is not applicable for targeting genes at will, as such a break can only be induced at the transgenic recognition sites of the rare cutting restriction enzyme, it can be used to excise transgenic sequences from the genome. Recently it could be demonstrated that a marker gene flanked by I-Scel sites and homologous sequences could be excised from transgenic tobacco plants. After I-Scel expression the marker gene was removed efficiently by HR as well as by NHEJ, demonstrating that at least DSB-induced recombination is a feasible alternative to site-specific recombinases for marker elimination (Siebert and Puchta 2002).

$\mathrm{HR}$ is for chloroplast and for bacteria and in contrast to the nuclei of higher eukaryotes the main mode of DNA recombination (Heifetz 2000, Bock 2001). Therefore, HR seems to be a suitable alternative for removing unwanted transgen sequences from the chloroplast genome. Recently this could be demonstrated by an elegant study in tobacco showing removal of different marker genes by the use of flanking homologies of 174 or 418 bps (lamtham and Day 2001).

\section{Conclusions}

A major goal of plant biotechnology is to improve existing and develop new elite cultivars. For this purpose, both improvement of existing and development of novel strategies for plant genome manipulation are required. To integrate a transgene at any specific genomic position in a controlled way and to remove transgene sequences are central issues in this context. Different approaches for elimination of selectable marker genes have been developed over the last years and further improvements are on the way. The techniques are in the process of being transferred to the crop plants of interest. Thus, there is no longer a need for bringing out transgenic plants of a new generation in the field that contain genes conferring antibiotic or herbicide resistance. Concerns about an uncontrolled spread of these genes in ecosystems will become obsolete in the near future. For gene targeting in higher plants however, the picture is still different. In spite of promising recent attempts it is not yet clear when and by which approach the final breakthrough will be achieved.

Acknowledgements. I thank Ingo Schubert for critical comments on the manuscript. The work in my laboratory is funded by grants of the Deutsche Forschungsgemeinschaft, the Kultusministerium of the Land Sachsen-Anhalt, the Bundesministerium für Bildung und Forschung; the German-Israel Foundation and the EU.

\section{References}

Albert H, Dale EC, Lee E, Ow DW (1995) Site-specific integration of DNA into wild-type and mutant lox sites placed in the plant genome. Plant J 7: 649-659

Arabidopsis Genome Initiative (2000) Analysis of the genome sequence of the flowering plant Arabidopsis thaliana. Nature 408: $796-815$

Ashton NW, Champagne CEM, Weiler T, Verkoczy LK (2000) The bryophyte Physcomitrella patens replicates extrachromosomal transgenic elements. New Phytol 146: 391-402

Bechtold N, Jaudeau B, Jolivet S, Maba B, Vezon D, Voisin R, Pelletier $G$ (2000) The maternal chromosome set is the target of the T-DNA in the in planta transformation of Arabidopsis thaliana. Genetics 155: 1875-1887

Beetham PR, Kipp PB, Sawycky XL, Arntzen CJ, May GD (1999) A tool for functional plant genomics: Chimeric RNA/DNA oligonucleotides cause in vivo gene-specific mutations. Proc Natl Acad Sci USA 96: 8774-8778

Bhatt AM, Canales C, Dickinson HG (2001) Plant meiosis: the means to $1 \mathrm{~N}$. Trends Plant Sci 6: 114-121

Bock R (2001) Transgenic plastids in basic research and plant biotechnology. J Mol Biol 312: 425-438

Carlson PS (1974) Mitotic crossing over in a higher plant. Genet Res Camb 24: 109-112

Cole-Strauss A, Gamper H, Holloman WK, Munoz M, Cheng N, Kmiec EB (1999) Targeted gene repair directed by the chimeric RNA/DNA oligonucleotide in a mammalian cell-free extract. Nucleic Acids Res 27: 1323-1330

Cole-Strauss A, Yoon K, Xiang Y, Byrne BC, Rice MC, Gryn J, Holloman WK, Kmiec EB (1996) Correction of the mutation responsible for sickle cell anemia by an RNA-DNA oligonucleotide. Science 273: $1386-1389$

Corneille S, Lutz K, Svab Z, Maliga P (2001) Efficient elimination of selectable marker genes from the plastid genome by the CRE-lox site-specific recombination system. Plant J 27: 171-178

Cove DJ, Knight CD, Lamparter T (1997) Mosses as model systems. Trends Plant Sci 2: 99-105

Dale EC, Ow DW (1991) Gene transfer with subsequent removal of the selection gene from the host genome. Proc Natl Acad Sci USA 23: 10558-10562 
De Block M, Debrouwer D (1991) Two T-DNAs co-transformed into Brassica napus by a double Agrobacterium infection are mainly integrated at the same locus. Theor Appl Genet 82: 257-263

Depicker A, Herman L, Jacobs S, Schell J, van Montagu M (1985) Frequencies of simultaneous transformation with different T-DNAs and their relevance to the Agrobacterium plant cell interaction. Mol Gen Genet 201: 477-484

Desfeux C, Clough SJ, Bent AF (2000) Female reproductive tissues are the primary target of Agrobacterium-mediated transformation by the Arabidopsis floral-dip method. Plant Physiol 123: 895-904

Dronkert ML, Beverloo HB, Johnson RD, Hoeijmakers JH, Jasin M, Kanaar R (2000) Mouse RAD54 affects DNA double-strand break repair and sister chromatid exchange. Mol Cell Biol 20: 3147-3156

Ebinuma H, Sugita E, Matsunaga E, Endo S, Yamada K, Komamine A (2001) Systems for the removal of a selection marker and their combination with a positive marker. Plant Cell Reports 20: 383-392

Ebinuma H, Sugita K, Matsunaga E, Yamakado M (1997) Selection of marker-free transgenic plants using the isopentenyl transferase gene. Proc Natl Acad Sci USA 94: 2117-2121

Engels WR (2000) Reversal of fortune for Drosophila geneticists? Science 288: 1973-1975

Gallego ME, Jeanneau M, Granier F, Bouchez D, Bechtold N, White I (2001) Disruption of the Arabidopsis RAD50 gene leads to plant sterility and MMS sensitivity. Plant J 25: 31-41

Gallego ME, Sirand-Pugnet P, White Cl (1999) Positive-negative selection and T-DNA stability in Arabidopsis transformation. Plant Mol Biol 39: 83-93

Gamper HB, Parekh H, Rice MC, Bruner M, Youkey H, Kmiec EB (2000) The DNA strand of chimeric RNA/DNA oligonucleotides can direct gene repair/conversion activity in mammalian and plant cellfree extracts. Nucleic Acids Res 28: 4332-4339

Gherbi H, Gallego ME, Jalut N, Lucht JM, Hohn B, White Cl (2001) Homologous recombination in planta is stimulated in the absence of Rad50. EMBO Rep 2: 287-291

Girke T, Schmidt H, Zahringer U, Reski R, Heinz E (1998) Identification of a novel delta 6-acyl-group desaturase by targeted gene disruption in Physcomitrella patens. Plant J 15: 39-48

Gleave AP, Mitra DS, Mudge SR, Morris BA (1999) Selectable markerfree transgenic plants without sexual crossing: transient expression of cre recombinase and use of a conditional lethal dominant gene. Plant Mol Biol 40: 223-235

Goldsbrough AP, Lastrella CN, Yoder JI (1993) Transposition mediated re-positioning and subsequent elimination of marker genes from transgenic tomato. Bio/technology 1993 11: 1286-1292

Gorbunova V, Levy AA (1999) How plants make ends meet: DNA double-strand break repair. Trends Plant Sci 4: 263-269

Gorbunova V, Levy AA (2000) Analysis of extrachromosomal Ac/Ds transposable elements. Genetics 155: 349-359

Gorbunova V, Avivi-Ragolski N, Shalev G, Kovalchuk I, Abbo S, Hohn B, Levy AA (2000) A new hyperrecombinogenic mutant of Nicotiana tabacum. Plant J 24: 601-611

Haber JE (1998) The many interfaces of Mre11. Cell 95: 583-586

Haldrup A, Petersen SG, Okkels FT (1998) The xylose isomerase gene from Thermoanaerobacterim thermosulfurogenes allows effective selection of transgenic plant cells using D-xylose as the selection agent. Plant Mol Biol 37: 287-296

Halfter U, Morris PC, Willmitzer L (1992) Gene targeting in Arabidopsis thaliana. Mol Gen Genet 231: 186-193
Hanin M, Mengiste T, Bogucki A, Paszkowski J (2000) Elevated levels of intrachromosomal homologous recombination in Arabidopsis overexpressing the MIM gene. Plant J 24: 183-189

Hanin M, Volrath S, Bogucki A, Bricker M, Ward E, Paszkowski J (2001) Gene targeting in Arabidopsis. Plant J 8: 671-677

Hansen G, Chilton MD (1999) Lessons in gene transfer to plants by a gifted microbe. Curr Top Microbiol Immunol 240: 21-57

Hartung F, Puchta $H$ (1999) Isolation of the complete cDNA of the Mre11 homologue of Arabidopsis (Accession No. AJ243822) indicates conservation of DNA recombination mechanisms between plants and other eucaryotes (PGR 99-132). Plant Physiol 121: 311

Hartung F, Plchova H, Puchta H (2000) Molecular characterization of RecQ homologues in Arabidopsis thaliana. Nucleic Acids Res 28: 4275-4282

Hajdukiewicz PT, Gilbertson L, Staub JM (2001) Multiple pathways for Cre/lox-mediated recombination in plastids. Plant J 27: 161-170

Heifetz PB (2000) Genetic engineering of the chloroplast. Biochimie 82: 655-666

Hohn B, Puchta H (1999) Gene therapy in plants. Proc Natl Acad Sci USA 96: 8321-8323

Hohn B, Levy AA, Puchta H (2001) Elimination of selection markers from transgenic plants. Curr Opin Biotech 12: 139-143

lamtham S, Day A (2001) Removal of antibiotic resistance genes from transgenic tobacco plastids. Nat Biotechnol 18: 1172-1176

Jasin M, Moynaham ME, Richardson C (1996) Targeted transgenesis. Proc Natl Acad Sci USA 93: 8804-8808

Joersbo M, Okkels T (1996) A novel principle for selection of transgenic plant cells: positive selection. Plant Cell Rep 16: 219-221

Joersbo M, Donaldson I, Kreiberg J, Petersen SG, Brundstedt J, Okkels FT (1998) Analysis of mannose selection used for transformation of sugar beet. Molecular Breeding 4: 111-117

Karow JK, Wu L, Hickson ID (2000) RecQ family helicases: roles in cancer and ageing. Curr Opin Genet Dev 10: 32-38

Kempin SA, Liljegren SJ, Block LM, Rounsley SD, Yanofsky MF, Lam E (1997) Targeted disruption in Arabidopsis. Nature 389: 802-803

Kilby NJ, Davies GJ, Snaith MR (1995) FLP recombinase in transgenic plants: constitutive activity in stably transformed tobacco and generation of marked cell clones in Arabidopsis. Plant J 8: 637-652

Komari T, Hiei Y, Saito Y, Murai N, Kumashiro T (1996) Vectors carrying two separate T-DNAs for co-transformation of higher plants mediated by Agrobacterium tumefaciens and segregation of transformants free from selection markers. Plant J 10: 165-174

Kumar S, Fladung M (2001) Controlling transgene integration in plants. Trends Plant Sci 6: 155-159

Kunkel T, Niu Q-W, Chan Y-S, Chua N-H (1999) Inducible isopentenyl transferase as a high efficiency marker for plant transformation. Nature Biotech 17: 916-919

Lee KY, Lund P, Lowe K, Dunsmuir P (1990) Homologous recombination in plant cells after Agrobacterium-mediated transformation. Plant Cell 2: $415-425$

Liljegren SJ, Yanofsky MF (1998) Towards targeted transformation in plants Response: targeting Arabidopsis. Trends Plant Sci 3: 79-80

Liu L, Rice MC, Kmiec EB (2001) In vivo gene repair of point and frameshift mutations directed by chimeric RNA/DNA oligonucleotides and modified single-stranded oligonucleotides. Nucleic Acids Res 29: 4238-4250

Lyznik LA, Rao KV, Hodges TK (1996) FLP-mediated recombination of FRT sites in the maize genome. Nucleic Acids Res 24: 3784-3789 
McKnight TD, Lillis MT, Simpson RB (1987) Segregation of genes transferred to one plant cell from two separate Agrobacterium strains. Plant Mol Biol 8: 439-445

Medberry SL, Dale E, Qin M, Ow DW (1995) Intra-chromosomal rearrangements generated by Cre-lox site-specific recombination. Nucleic Acids Res 23: 485-490

Mengiste T, Paszkowski J (1999) Prospects for the precise engineering of plant genomes by homologous recombination. Biol Chem 380: 749-758

Mengiste T, Revenkova E, Bechtold N, Paszkowski J (1999) An SMClike protein is required for efficient homologous recombination in Arabidopsis. EMBO J 18: 4505-4512

Miao Z-H, Lam E (1995) Targeted disruption of the TGA3 locus in Arabidopsis thaliana. Plant J 7: 359-365

Negrotto D, Jolley M, Beer S, Wenck AR, Hansen G (2000) The use of phosphomannose-isomerase as a selectable marker to recover transgenic maize plants (Zea mays L.) via Agrobacterium transformation. Plant Cell Rep 19: 798-803

Offringa R, de Groot MJA, Haagsman HJ, Does MP, van den Elzen PJM, Hooykaas PJJ (1990) Extrachromosomal homologous recombination and gene targeting in plant cells after Agrobacterium mediated transformation. EMBO J 9: 3077-3084

Offringa R, Franke-van Dijk MEI, de Groot MJA, van den Elzen PJM, Hooykaas PJJ (1993) Nonreciprocal homologous recombination between Agrobacterium transferred DNA and a plant chromosomal locus. Proc Natl Acad Sci USA 90: 7346-7350

Oh TJ, May GD (2001) Oligonucleotide-directed plant gene targeting. Curr Opin Biotechnol 12: 169-172

Onouchi H, Nishihama R, Kudo M, Machida Y, Machida C (1995) Visualization of site-specific recombination catalyzed by a recombinase from Zygosaccharomyces rouxii in Arabidopsis thaliana. Mol Gen Genet 247: 653-660

Osborne BI, Wirtz U, Baker B (1995) A system for insertional mutagenesis and chromosomal rearrangement using the Ds transposon and Cre-lox. Plant J 7: 687-701

Ow DW (1996) Recombinase-directed chromosome engineering in plants. Cur Op Biotechnol 7: 181-186

Ow DW (2001) The right chemistry for marker gene removal? Nat Biotechnol 19: 115-116

Ow DW (2002) Applications of site-specific recombination. Plant Mol Biol 48: 183-200

Paques F, Haber JE (1999) Multiple pathways of recombination induced by double-strand breaks in Saccharomyces cerevisiae. Microbiol Mol Biol Rev 63: 349-404

Paszkowski J, Baur M, Bogucki A, Potrykus I (1988) Gene targeting in plants. EMBO J 7: 4021-4026

Potrykus I, Spangenberg G (1995) Gene transfer to plants. Springer, Berlin

Puchta H (1998 a) Towards targeted transformation in plants. Trends Plant Sci 3: 77-78

Puchta H (1998b) Repair of genomic double-strand breaks in somatic plant cells by one-sided invasion of homologous sequences. Plant J 13: 331-339

Puchta H (1999) DSB-induced recombination between ectopic homologous sequences in somatic plant cells. Genetics 152: 1173-1181

Puchta H (2000) Removing selectable marker genes: taking the shortcut. Trends in Plant Sci 5: 273-274

Puchta H (2002) Gene replacement by homologous recombination in plants. Plant Mol Biol 48: 173-182
Puchta H, Hohn B (1996) From cent Morgans to basepairs: Homologous recombination in plants. Trends in Plant Sci 1: 340-348

Puchta H, Dujon B, Hohn B (1996) Two different but related mechanisms are used in plants for the repair of genomic double-strand breaks by homologous recombination. Proc Natl Acad Sci USA 93: 5055-5060

Qin M, Bayley C, Stockton T, Ow DW (1994) Cre recombinasemediated site-specific recombination between plant chromosomes. Proc Natl Acad Sci USA 91: 1706-1710

Reiss B, Klemm M, Kosak H, Schell J (1996) RecA protein stimulates homologous recombination in plants. Proc Natl Acad Sci USA 93: 3094-3098

Reiss B, Schubert I, Köpchen K, Wendeler E, Schell J, Puchta H (2000) RecA stimulates sister chromatid exchange and the fidelity of double-strand break repair, but not gene targeting, in plants transformed by Agrobacterium. Proc Natl Acad Sci USA 97: 33583363

Reski R (1998 a) Development, genetics and molecular biology of mosses. Bot Acta 111: 1-15

Reski R (1998 b) Physcomitrella and Arabidopsis: the David and Goliath of reverse genetics. Trends Plant Sci 3: 209-210

Reski R (1999) Molecular genetics of Physcomitrella. Planta 208: 301309

Reski R, Reynolds S, Wehe M, Kleber-Janke T, Kruse S (1998) Moss (Physcomitrella patens) expressed sequence tags include several sequences which are novel for plants. Bot Acta 111: 145-151

Rice MC, May GD, Kipp PB, Parekh H, Kmiec EB (2000) Genetic repair of mutations in plant cell-free extracts directed by specific chimeric oligonucleotides. Plant Physiol 123: 427-438

Rice MC, Czymmek K, Kmiec EB (2001) The potential of nucleic acid repair in functional genomics. Nat Biotechnol 19: 321-326

Risseeuw E, Franke-van Dijk ME, Hooykaas PJ (1997) Gene targeting and instability of Agrobacterium T-DNA loci in the plant genome. Plant J 11: 717-728

Risseeuw E, Offringa R, Franke-van Dijk ME, Hooykaas PJ (1995) Targeted recombination in plants using Agrobacterium coincides with additional rearrangements at the target locus. Plant J 7: 109-119

Rong YS, Golic KG (2000) Gene targeting by homologous recombination in Drosophila. Science 288: 2013-2018

Rong YS, Golic KG (2001) A targeted gene knockout in Drosophila. Genetics 157: 1307-1312

Rossi L, Tinland B, Hohn B (1996) Role of the virulence protein of Agrobacterium tumefaciens in the plant cell. In: Spaink H, Hooykaas P, Kondorosi A (eds) The Rhizobiaceae. Kluwer, Dordrecht, pp 303-320

Rubin E, Levy AA (1997) Abortive gap repair: The underlying mechanism for Ds elements formation. Mol Cell Biol 17: 6294-6302

Russell SH, Hoopes JL, Odell JT (1992) Directed excision of a transgene from the plant genome. Mol Gen Genet 234: 49-59

Salomon S, Puchta H (1998) Capture of genomic and T-DNA sequences during double-strand break repair in somatic plant cells. EMBO J 17: 6086-6095

Schaefer DG (2001) Gene targeting in Physcomitrella patens. Curr Opin Plant Biol 4: 143-150

Schaefer DG, Zryd JP (1997) Efficient gene targeting in the moss Physcomitrella patens. Plant J 11: 1195-1206

Shalev G, Sitrit Y, Avivi-Ragolski N, Lichtenstein C, Levy AA (1999) Stimulation of homologous recombination in plants by expression of the bacterial resolvase RuvC. Proc Natl Acad Sci USA 96: 7398 7402 
Siebert R, Puchta H (2002) Efficient repair of genomic double-strand breaks by homologous recombination between directly repeated sequences in the plant genome. Plant Cell 14: 1121-1131

Srivastava V, Anderson OD, Ow DW (1999) Single-copy transgenic wheat generated through the resolution of complex integration patterns. Proc Natl Acad Sci USA 96: 11117-11121

Strepp R, Scholz S, Kruse S, Speth V, Reski R (1998) Plant nuclear gene knockout reveals a role in plastid division for the homolog of the bacterial cell division protein FtsZ, an ancestral tubulin. Proc Natl Acad Sci USA 95: 4368-4373

Sugita K, Kasahara T, Matsunaga E, Ebinuma H (2000) A transformation vector for the production of marker-free transgenic plants containing a single copy transgene at high frequency. Plant J 22: 461469

Swoboda P, Gal S, Hohn B, Puchta H (1994) Intrachromosomal homologous recombination in whole plants. EMBO J 13: 484-489

Thomason LC, Calendar R, Ow DW (2001) Gene insertion and replacement in Schizosaccharomyces pombe mediated by the Streptomyces bacteriophage phiC31 site-specific recombination system. Mol Genet Genomics 265: 1031-1038

Thykjaer T, Finnemann J, Schauser L, Christensen L, Poulsen C, Stougaard J (1997) Gene targeting approaches using positive-negative selection and large flanking regions. Plant Mol Biol 35: 523-530

Tinland B (1996) The integration of T-DNA into plant genomes. Trends Plant Sci 1: 178-184

Van der Steege G, Schuilenga-Hut PH, Buys $\mathrm{CH}$, Scheffer $\mathrm{H}$, Pas $\mathrm{HH}$, Jonkman MF (2001) Persistent failures in gene repair. Nat Biotechnol 19: 305-306

Vergunst AC, Hooykaas PJJ (1999) Recombination in the plant genome and its application in biotechnology. Crit Rev Plant Sci 18: $1-31$

Vergunst AC, Jansen LE, Hooykaas PJ (1998) Site-specific integration of Agrobacterium T-DNA in Arabidopsis thaliana mediated by Cre recombinase. Nucleic Acids Res 26: 2729-2734

Wang W, Seki M, Narita Y, Sonoda E, Takeda S, Yamada K, Masuko T, Katada T, Enomoto T (2000) Possible association of BLM in decreasing DNA double strand breaks during DNA replication. EMBO J 19: 3428-3435
West CE, Waterworth WM, Jiang Q Bray CM (2000) Arabidopsis DNA ligase IV is induced by gamma-irradiation and interacts with an Arabidopsis homologue of the double strand break repair protein XRCC4. Plant J 24: 67-78

Xiaohui Wang H, Viret J, Eldridge A, Perera R, Signer ER, Chiurazzi M (2001) Positive-Negative selection for homologous recombination in Arabidopsis. Gene 272: 249-255

Yamaguchi-Iwai Y, Sonoda E, Sasaki MS, Morrison C, Haraguchi T, Hiraoka Y, Yamashita YM, Yagi T, Takata M, Price C, Kakazu N, Takeda S (1999) Mre11 is essential for the maintenance of chromosomal DNA in vertebrate cells. EMBO J 18: 6619-6629

Ye S, Cole-Strauss AC, Frank B, Kmiec EB (1998) Targeted gene correction: a new strategy for molecular medicine Mol Med Today 4 : 431-437

Ye GN, Stone D, Pang SZ, Creely W, Gonzalez K, Hinchee M (1999) Arabidopsis ovule is the target for Agrobacterium in planta vacuum infiltration transformation. Plant J 19: 249-257

Yoder JI, Goldsbrough AP (1994) Transformation systems for generating marker-free transgenic plants. Bio/technology 12: 263-267

Zhu T, Peterson DJ, Tagliani L, St Clair G, Baszczynski C, Bowen B (1999) Targeted manipulation of maize genes in vivo using chimeric RNA/DNA oligonucleotides. Proc Natl Acad Sci USA 96: 8768-8773

Zhu T, Mettenburg K, Peterson DJ, Tagliani L, Baszczynski CL (2000) Engineering herbicide-resistant maize using chimeric RNA/DNA oligonucleotides. Nat Biotechnol 18: 555-558

Zubko E, Scutt C, Meyer P (2000) Intrachromosomal recombination between attP regions as a tool to remove selectable marker genes from tobacco transgenes. Nat Biotechnol 18: 442-445

Zuo J, Niu QW, Moller SG, Chua NH (2001) Chemical-regulated, sitespecific DNA excision in transgenic plants. Nat Biotechnol 19: 157161

Zupan J, Muth TR, Draper O, Zambryski P (2000) The transfer of DNA from Agrobacterium tumefaciens into plants: a feast of fundamental insights. Plant J 23: 11-28 
Karlsruher Institut für Technologie

\section{Repository KITopen}

Dies ist ein Postprint/begutachtetes Manuskript.

Empfohlene Zitierung:

Puchta, $\mathrm{H}$.

Towards the ideal GMP: Homologous recombination and marker gene excision. 2003. Journal of plant physiology, 160

doi:10.5445/IR/1000016584

Zitierung der Originalveröffentlichung:

Puchta, $\mathrm{H}$.

Towards the ideal GMP: Homologous recombination and marker gene excision. 2003. Journal of plant physiology, 160, 743-754.

doi:10.1078/0176-1617-01027 